\title{
Actualidad institucional y económica de España en el marco de la Unión Europea (Enero 2020)
}

\author{
Beatriz Iñarritu \\ Profesora de la 'Deusto Business School' \\ Universidad de Deusto
}

doi: http://dx.doi.org/10.18543/ced-62-2020pp227-242

Sumario: I. Introducción- II. El Estado de la Integración- III. Cuestiones generales de la actualidad económica

\section{Introducción}

Durante el segundo semestre de 2019 se ha producido un importante cambio de ciclo en las instituciones de la UE, con el inicio de la novena legislatura del Parlamento Europeo y el nombramiento de una nueva Comisión y de los nuevos presidentes del Consejo Europeo y del Banco Central Europeo. Los retos a los que deberán enfrentarse todos ellos no son menores; el Brexit, la desaceleración económica, la emergencia climática o la agenda digital entre otros.

La designación de los nuevos líderes nos deja un balance de preponderancia franco-alemana puesto que estos dos países han conseguido los puestos más relevantes en juego, la presidencia de la Comisión y del Banco Central, aunque ciertamente es un balance inusual puesto que ambos puestos han sido ocupados por primera vez en la historia por dos mujeres.

Por su parte, la parálisis del Brexit parece tener sus días contados tras el mayoritario apoyo otorgado por los británicos al nuevo primer ministro Boris Johnson, quien podrá hacerlo efectivo el 31 de Enero sobre la base del acuerdo de salida pactado en Noviembre.

También fueron cuestiones importantes en el semestre las nuevas medidas expansivas de política monetaria anunciadas por Mario Draghi apenas un mes antes de abandonar la presidencia del BCE y los nuevos aranceles de Estados Unidos sobre productos europeos tras la decisión de la OMC por las ayudas públicas concedidas a Airbus.

During the second six months of 2019 an important change has occurred in the EU institutions, with the start of the ninth term of office of the European Parliament, and the nomination of the new Commission and 
presidents of the European Council and European Central Bank. The challenges they will have to face are not minor ones; the Brexit, the economic deceleration, the climate emergency or the digital agenda, among others.

The appointment of the new leaders gives us a balance of French and German preponderance as these two countries have achieved the most relevant post at stake, both presidencies of the Commission and the Central Bank, although it is actually a very unusual result as for the first time in history two women were assuming these responsibilities.

On the other hand, the paralysis provoked by the Brexit is coming to an end after the major support given by the British citizens to the new prime minister Boris Johnson, who will be able to make it effective on January $31^{\text {st }}$ on the grounds of the new exit agreement concluded in November.

Two other questions were relevant in this semester, the new expansive measures of monetary policy announced by Mario Draghi just one month before leaving the presidency of the ECB and the new customs duties implemented by the United States on European products after the WTO decision on the public aids given to Airbus.

\section{El estado de la integración}

\section{La nueva Comisión Von der Leyen 2019-2024}

En el Consejo Europeo celebrado el 2 de Julio la ministra de Defensa alemana, Ursula Von der Leyen, fue designada para presidir la Comisión Europea, lo que la convertiría en la primera mujer de la historia en dirigir el ejecutivo europeo.

El acuerdo incluía, también, la propuesta de Josep Borrell, ministro de Exteriores español en funciones, para el cargo de vicepresidente de la Comisión y alto representante de Política Exterior de la UE.

La elección de la alemana se produjo tras el fracaso del llamado proceso de Spitzenkandidaten (candidatos a la presidencia de la Comisión elegidos por los partidos políticos), ya que tanto el popular Manfred Weber como el socialista Frans Timmermans, cabezas de lista en las elecciones europeas, fueron apartados de la candidatura y se designó a una política como Von der Leyen que ni tan siquiera había participado en las elecciones europeas.

Este pulso de los Estados miembros sobre al Parlamento Europeo no evitó que. en su sesión plenaria del 16 de Julio, la Eurocámara ratificara el nombramiento de Von der Leyen, aunque ciertamente por un estrecho margen puesto que tan sólo consiguió el respaldo de 383 diputados, apenas una decena de votos más que la mayoría absoluta imprescindible (376) y siendo éste un apoyo muy inferior al que había logrado su antecesor en el puesto, Jean-Claude Juncker, cinco años atrás. 
En su intervención ante el pleno del Parlamento, Von der Leyen planteó la posibilidad de desarrollar diferentes iniciativas como un marco legal para establecer un salario mínimo a escala comunitaria, un seguro europeo de desempleo complementario a los nacionales o el impulso de un green deal (pacto verde) en sus primeros 100 días de gobierno, promoviendo inversiones sostenibles por un billón de euros.

Y tampoco faltaron alusiones a su compromiso a favor de la igualdad de género, sobre una mayor flexibilidad en el Pacto de Estabilidad y Crecimiento, o sobre la necesidad de revisar la política migratoria de la UE.

Tras la presentación de las candidaturas de Von der Leyen y Borrell, el proceso de nombramiento del resto de comisarios continuó con las propuestas del resto de Estados miembros, hasta que finalmente, el 27 de Noviembre, el pleno de la Eurocámara dio de nuevo su apoyo al conjunto de candidatos a ocupar los puestos de comisarios (27 candidatos, ya que el Reino Unido renunció a presentar su propuesta).

De esta forma, el nuevo ejecutivo comunitario, destinado a desarrollar su mandato durante los siguientes cinco años, consiguió el apoyo de 461 diputados procedentes de los grupos popular, socialdemócrata y liberal del Parlamento Europeo (eran necesarios al menos 376 votos sobre 751), frente a 157 votos en contra y 89 abstenciones. Y así fue como, tras esta «sesión de investidura», los nuevos comisarios pudieron tomar posesión de sus cargos el 2 de Diciembre, un mes más tarde de lo previsto,

El retraso en este inicio del mandato de la nueva Comisión se debió al rechazo mostrado por el Parlamento Europeo respecto de tres candidatos, los propuestos por Francia, Rumanía y Hungría.

En efecto, en Octubre se había producido el insólito rechazo de la candidata francesa, la liberal Sylvie Goulard, en las audiencias previas ante el Parlamento, a pesar de que nunca antes se había rechazado a un candidato francés al puesto de comisario. Este veto fue interpretado en diferentes medios como una «revancha política» del Partido Popular Europeo contra el presidente francés, Emmanuel Macron, puesto que fue quien había liderado en Junio la campaña para impedir que el conservador alemán, Manfred Weber, se alzara con la presidencia de la Comisión Europea.

La francesa no logró despejar las dudas planteadas sobre su integridad y sus posibles conflictos de interés en el pasado, y las comisiones parlamentarias ante las que intervino pusieron el énfasis en los puntos más oscuros de su currículum: la investigación abierta en Francia por irregularidades en el pago de asistentes parlamentarios y su trabajo para un centro de estudios estadounidense durante su etapa como eurodiputada.

De esta forma, París, lo mismo que Bucarest y Budapest, tuvo que proponer un nuevo candidato que, efectivamente, ofreciera mayores garantías de recibir el apoyado de la Cámara europea. 
En su nuevo discurso de Noviembre ante el pleno del Parlamento Europeo en Estrasburgo, la presidenta electa de la Comisión presentó, junto a su equipo, el que iba a ser su programa político, mencionando como temas prioritarios de su mandato el cambio climático, la digitalización, los fenómenos migratorios y la igualdad de género.

Y se explayó en particular en las dos áreas que están llamadas a ser la clave de las políticas de la nueva Comisión: el Green Deal, que deberá liderar el laborista holandés Frans Timmermans, y la agenda digital, cuyas gestión será asumida por la liberal danesa Margrethe Vestager.

Atrás quedaba también, la polémica desatada a comienzos de Noviembre por la denominación de una de las carteras de la nueva Comisión. El anuncio de que el comisario griego Margaritis Schinas iba a ser responsable de las cuestiones relacionadas con la emigración bajo el título de «protección del modo de vida europeo» provocó una crítica inmediata desde distintos ámbitos, por lo que Von der Leyen admitió finalmente el cambio del término «protección» por el de «promoción» del modo de vida europeo.

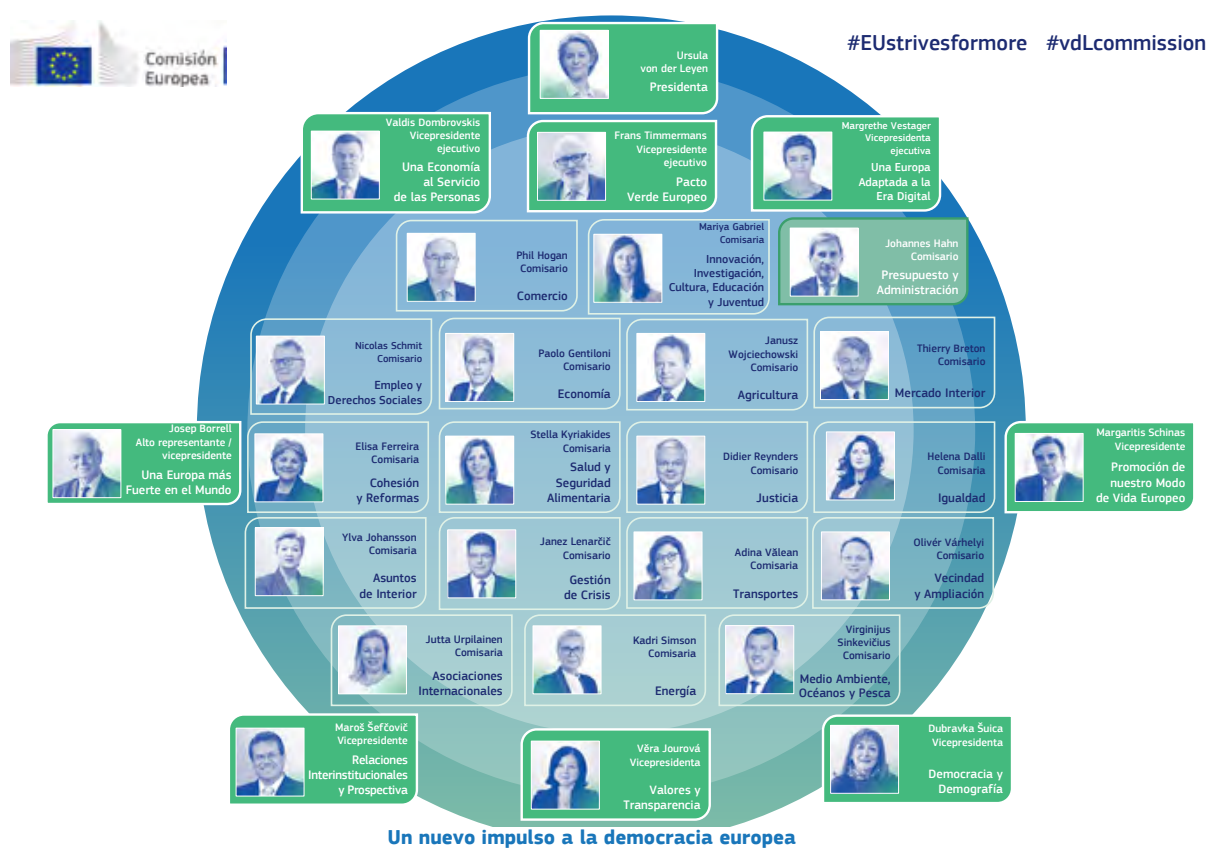

Fuente: Comisión Europea

https://ec.europa.eu/commission/commissioners/sites/comm-cwt2019/files/team_attachments/ globe-college-protocol-2019-2024_es.pdf 


\section{Consejo Europeo: Charles Michel nuevo presidente}

El mismo Consejo Europeo celebrado el 2 de Julio eligió a su nuevo presidente, Charles Michel, para un período comprendido entre el 1 de Diciembre de 2019 y el 31 de Mayo de 2022, en sustitución del polaco Donald Tusk. Su mandato sería, por tanto, de dos años y medio, siendo renovable una sola vez.

De manera simultánea, los Jefes de Estado o de Gobierno de los Estados miembros cuya moneda es el euro, es decir, de la Eurozona, también decidieron que Michel se convirtiera, por el mismo período de tiempo, en presidente de esta Cumbre del Euro.

Michel era entonces primer ministro belga en funciones y en medios políticos se reconocía su particular habilidad para liderar equilibrios imposibles, ya que durante casi cuatro años había sido capaz de mantener a flote en Bélgica un gobierno formado por una difícil coalición cuatripartita.

En su primera rueda de prensa tras ser elegido para liderar el Consejo Europeo, Michel puso en valor los dos principios que, según su criterio, habían guiado su gobierno, unidad y diversidad, y también destacó la base fundamental de una intensa «dosis de diálogo». Declaró, en clara referencia a la nueva responsabilidad que asumía que «la diversidad no tiene por qué ser fragilidad».

\section{BCE: nombramiento de la nueva presidenta Christine Lagarde}

Y también el Consejo Europeo de Julio anunció el consenso alcanzado respecto al nombramiento de la sustituta de Mario Draghi al frente del Banco Central Europeo en el momento en que el italiano finalizara su mandato el 31 de Octubre.

La elegida fue la francesa Christine Largade, la entonces directora gerente del Fondo Monetario Internacional, FMI, y que, al igual que sucedía con Von der Leyen en la Comisión Europea, iba a convertirse en la primera mujer en presidir la autoridad monetaria de la Eurozona, lo que significaba un paso más en la paridad de género de las instituciones comunitarias.

En todo caso, la nominación de Lagarde suscitó cierta sorpresa por su inexperiencia en lo que respecta a la política monetaria, a pesar de que es cierto que la propia normativa del BCE permite acceder al cargo a candidatos con experiencia en el sector financiero, algo que ya sirvió a Luis de Guindos para hacerse con la vicepresidencia.

Y por otra parte, también se escucharon opiniones sobre el hecho de que con su nombramiento se neutralizaban las aspiraciones del alemán 
Jens Weidmann, gobernador del Banco de Alemania, un «halcón» monetario temido por Francia, Italia o España; el nombramiento de la francesa, presumiblemente más favorable a una política monetaria relajada, tranquilizaba, en particular, al gobierno de Italia, ya que este país muestra un desequilibrio importante en sus cuentas públicas con una deuda pública equivalente al $135 \%$ de su PIB.

En sus años al frente del Fondo Monetario Internacional (FMI), Lagarde ya mostró, en diferentes ocasiones, su postura a favor de estímulos monetarios extraordinarios en época de crisis, por lo que, en los mercados financieros, es considerada como una «paloma» (dove, en la jerga), es decir, como una política con querencia por los tipos bajos para estimular la economía.

Lagarde se enfrentará a la difícil tarea de reemplazar a un Draghi a quien se reconoce como «el salvador del euro» y que es recordado en particular por su famoso discurso de Londres, en el que en plena crisis de deuda soberana prometió hacer «lo que sea necesario» («whatever it takes»). Una de las principales misiones de la francesa será, efectivamente, demostrar determinación y defender la credibilidad ya conseguida por el BCE.

4. Parlamento Europeo: nuevo presidente, David Sassoli, y reducción del número de escaños con el Brexit

La novena legislatura del Parlamento Europeo se puso en marcha tras la celebración de las elecciones de Mayo.

Y en el pleno celebrado el 3 de Julio, los eurodiputados eligieron al italiano David Sassoli como presidente del Parlamento Europeo, con 345 votos favorables de los 667 emitidos en la segunda ronda de votación, para ocupar este mandato hasta Enero de 2022, el equivalente a la mitad de la legislatura, en sustitución del también italiano, Antonio Tajani.

Eurodiputado desde 2009 y vicepresidente de esta institución durante la octava legislatura, Sassoli había concurrido a las elecciones de Mayo en las listas del Partido socialdemócrata europeo, S\&D.

Su nombramiento fue posible gracias a los sufragios de su grupo, pero también a los de liberales y populares, quienes rehusaron presentar candidatos a la presidencia de la Eurocámara a cambio de hacerse con otros cargos en el reparto de poder en las instituciones europeas.

En su primera intervención ante el pleno tras su elección, el italiano señaló que »en estos meses, demasiada gente ha azuzado divisiones y conflictos que nos traen malos recuerdos de la historia. Los ciudada- 
nos, por su parte, han demostrado que siguen creyendo en este proyecto, el único capaz de ofrecer respuestas a los desafíos globales que afrontamos». Y citó, entre las prioridades del Parlamento Europeo para los próximos años, el desempleo juvenil, la migración, el cambio climático, la revolución digital y el nuevo equilibrio mundial.

Por otra parte, la inminente salida británica de la UE significará un cambio en la composición del Parlamento, ya que los 73 eurodiputados del Reino Unido dejarán su escaño en Febrero al consumarse el Brexit. Un total de 46 de esos puestos se eliminarán, dejando el hemiciclo con 705 eurodiputados. El resto se repartirán entre varios Estados miembros, entre ellos, España, que pasará de 54 a 59 asientos.

\section{Brexit: nuevo acuerdo de salida de la UE y prórroga hasta el 31 de Enero}

Tras el anuncio de un segundo aplazamiento, hasta el 31 de Octubre, para llevar a cabo la salida del Reino Unido de la UE y tras la dimisión de Theresa May hecha pública el 24 de Mayo, los conservadores británicos eligieron a Boris Johnson como nuevo primer ministro del Reino Unido en Julio.

Ante los militantes tories que mayoritariamente le habían dado su apoyo, Johnson se reiteró en su intención de llevar a cabo el Brexit y de demostrar «su habilidad para equilibrar dos instintos enfrentados: el deseo de mantener una relación cercana con la UE y el deseo de que el Reino Unido pueda autogobernarse democráticamente».

Johnson debía, presumiblemente, asumir el acuerdo ya firmado por May con la UE en Noviembre de 2018 y que incluía el llamado backstop (la salvaguarda irlandesa), según el cual Irlanda del Norte se mantendría sine die en el territorio aduanero de la Unión Europea (hasta la conclusión del futuro acuerdo comercial del Reino Unido con la UE), para evitar una nueva frontera entre las dos Irlandas, la república y la región británica de Irlanda del Norte, pero que, por otra parte, provocaría la ruptura de la integridad territorial del Reino Unido.

Esta segunda circunstancia fue el factor determinante para que, en efecto, Johson, anunciara de manera inmediata su determinación para renegociar el pacto negociado por su predecesora, a pesar de la resistencia declarada por la mayoría de Estados comunitarios.

De hecho, y con la idea de forzar esta renegociación, Johnson no ocultó la posibilidad de que el Reino Unido pudiera abandonar la UE sin acuerdo, a pesar de no contar con una mayoría suficiente en el Parlamento británico. En diferentes votaciones los diputados británicos de la oposi- 
ción, y también varias decenas de conservadores moderados, mostraron su frontal oposición a este Brexit «duro».

En Agosto, Johnson pidió y obtuvo de la reina Isabel el permiso para suspender la actividad del Parlamento, una maniobra que pretendía restar tiempo a la oposición para frenar la posibilidad de un Brexit sin acuerdo, y que fue calificada por muchos medios políticos como un «escándalo constitucional». El dictamen emitido por el Tribunal Supremo británico sirvió para acortar este período de inactividad parlamentaria que, finalmente, se prolongó durante quince días, entre el 10 y el 25 de Septiembre.

El 2 de Octubre Johnson remitía al presidente de la Comisión Europea, Jean-Claude Juncker, su nueva propuesta de acuerdo de salida de la UE que dejaba fuera la pieza más incómoda, el «backstop» o cláusula de salvaguardia para Irlanda de Norte, con la incorporación de este territorio a la Unión Aduanera del Reino Unido pero garantizando en todo caso que, tras el Brexit, no se establecería una frontera física entre las dos Irlandas.

La no imposición de un control aduanero en el interior de la isla permitiría mantenerla como una unidad económica con movimientos fluidos y sin esperas en el tráfico comercial, aunque la pertenencia de Irlanda del Norte al territorio aduanero británico obligaría a establecer barreras aduaneras, con controles telemáticos sobre las mercancías alejados de la frontera terrestre, en el mar, entre Irlanda del Norte y el resto del Reino Unido.

Según lo expuesto por el premier británico, los controles aduaneros se reducirían al mínimo gracias a un nuevo sistema de notificación y seguimiento de las mercancías. Y aunque Johnson admitía que «los controles físicos seguirán siendo necesarios, éstos se efectuarían en una muy pequeña proporción». Señalaba que se podrían llevar a cabo «en las dependencias de las empresas exportadoras o en otros lugares que podrían estar localizados en cualquier parte de Irlanda o de Irlanda del Norte».

Johnson minimizaba el impacto de esta cuestión subrayando que el comercio de bienes entre las dos partes de Irlanda «es poco más del $1 \%$ del comercio total entre Reino Unido y la UE», por lo que, en su opinión, podría ser razonable gestionar esta frontera de manera diferente.

Las negociaciones llevadas a cabo entre Londres y Dublín, lideradas por los dos primeros ministros, Boris Johnson y Leo Varadkar, parecían abrir el camino hacia la consecución del acuerdo, lo que facilitó que, en efecto, el Consejo Europeo celebrado el 17 de Octubre anunciara su aprobación del nuevo acuerdo sobre el Brexit.

El nuevo pacto alcanzado entre Bruselas y Londres, trataría, en efecto, de evitar una frontera dura en el interior de la isla de Irlanda a 
pesar de que Irlanda del Norte formaría parte de la Unión Aduanera del Reino Unido, aunque cumpliendo diferentes normas relativas al Mercado Único Europeo.

Este protocolo significaría que las fronteras en las que deberían realizarse los controles y verificaciones sobre los productos deberían situarse en el mar de Irlanda y no en el interior de la isla. Los controles necesarios serían realizados en las zonas portuarias del mar de Irlanda por parte de las autoridades británicas y serían supervisados por la UE.

De este modo, las verificaciones que deban realizarse a los productos británicos que quieran exportarse a la UE a través de Irlanda se harían cuando entraran en Irlanda del Norte y no en la frontera entre este territorio británico y la República de Irlanda.

En todo caso, el acuerdo otorgaba un papel destacado a la Asamblea de Irlanda del Norte, puesto que gracias a un «mecanismo de consentimiento», este Parlamento regional podría, tras los primeros cuatro años de aplicación del protocolo, decidir si éste sigue en vigor o no.

Sin embargo, y dado que los diputados del Parlamento británico rechazaron llevar a cabo la votación sobre el nuevo acuerdo del Brexit con la urgencia necesaria para culminarlo en la fecha prevista del 31 de Octubre, Johnson se vio obligado a solicitar una tercera prórroga en la ejecución del Brexit, con un plazo extra de tres meses, hasta el 31 de Enero de 2020.

El gobierno británico se vio forzado a aparcar la tramitación legislativa del acuerdo y, finalmente, consiguió el 29 de Octubre que el Parlamento accediera a la celebración de elecciones generales anticipadas en el país, como única solución posible al laberinto y a la parálisis del Brexit.

El resultado de las elecciones, que se celebraron el 12 de Diciembre no dejó ninguna duda sobre la voluntad de los británicos sobre el Brexit, ya que Boris Johnson logró una de las mayores victorias del Partido Conservador de las últimas décadas. Los tories consiguieron la mayoría absoluta en el Parlamento con 365 escaños (frente a los 317 logrados por Theresa May en 2017), mientras que los laboristas solo consiguieron 203, su peor dato desde 1935.

Muchos analistas destacaron el voto recibido por Johnson de millones de británicos de las regiones del norte de Inglaterra de tradicional voto laborista para apoyar el mensaje conservador centrado en una idea clave: «Get Brexti done» (hagamos realidad el Brexit).

De esta forma, se allanaba el camino hacia el divorcio, puesto que el apoyo mayoritario del nuevo Parlamento permitiría al gobierno de Johnson acabar con la parálisis política en la que Reino Unido se encuentra desde Junio de 2016. 
Johnson no tuvo ninguna dificultad en recibir, por fin, un holgado apoyo parlamentario el 20 de Diciembre, 358 votos a favor y 234 en contra, para llevar a cabo el nuevo acuerdo de salida de la UE.

En todo caso, este nuevo proyecto de ley incorporaba ciertas novedades importantes respecto al que negoció Johnson con la UE en Octubre, como la prohibición de pactar nuevas prórrogas al Brexit y la limitación del periodo de transición al ya conocido 31 de Diciembre de 2020.

De esta forma, una vez que el Reino Unido abandone la UE el 31 de Enero de 2020, se presenta el nuevo reto de negociar la futura relación comercial y conseguir cerrar un acuerdo antes de que acabe al año, plazo considerado del todo insuficiente en medios comunitarios.

\section{Unión Económica y Monetaria: avances en el presupuesto para la Eurozona y retrasos en la reforma del MEDE y en la creación del FUGD}

En Octubre los ministros de Economía y Finanzas del Eurogrupo alcanzaron nuevos acuerdos en relación al futuro presupuesto de la Eurozona, conocido oficialmente como el BICC, por las siglas en inglés de «instrumento presupuestario para la convergencia y la competitividad».

A pesar de su modesta dotación inicial (se baraja una cifra de 17.000 millones de euros para el período 2021-27), sería previsible que este nuevo instrumento financiero reservara un $20 \%$ de los fondos (unos 3.400 millones) para «reaccionar a los retos específicos de cada país», es decir, para afrontar situaciones de crisis, lo que, sin duda, satisface las principales demandas que habían planteado los gobiernos de España y Francia.

Además, el acuerdo también recogía la reivindicación española sobre que los países en dificultades puedan dedicar los fondos del Presupuesto de la UME a inversiones y reformas sin más condiciones que las exigidas para acceder a otros fondos. De esta forma, la recepción de fondos del Presupuesto de la Eurozona no supondría la imposición de una «condicionalidad» similar a la que se impone en los casos de los rescates.

El comisario de Asuntos Económicos, Pierre Moscovici, señaló que, dado que se trata de un instrumento todavía modesto, deberán darse nuevos pasos hacia la creación de un auténtico presupuesto encaminado a tener una verdadera función de estabilización.

En cambio, en su reunión de Diciembre el Eurogrupo no consiguió avances relevantes respecto al refuerzo del Mecanismo Europeo de Estabilidad, MEDE, y sobre la creación del Fondo Único de Garantía de Depósitos, FUGD. 
El principal escollo para reformar el MEDE vino de Roma, por la oposición mostrada por el gobierno italiano respecto a las llamadas «cláusulas de acción colectiva», que pretenden facilitar los procesos de reestructuración de la deuda soberana impidiendo que un accionista minoritario pueda bloquear estos procesos en contra de los mayoritarios. Con una deuda del $135 \%$ sobre su PIB, los críticos en Italia con esa cláusula temen que, en efecto, su inclusión pudiera llevar a una reestructuración de su deuda de manera inevitable.

De esta forma, y a pesar de los meses de negociación ya dedicados a la reforma del MEDE, los demás países del Euro no consiguieron vencer la negativa de Roma y lo que debía haber sido un pacto definitivo sobre el MEDE reformado se quedó, aún, en un principio de acuerdo.

Y por lo que se refiere al FUGD, y a pesar de que la reunión de Noviembre había finalizado con cierto optimismo tras producirse del desbloqueo por parte de Alemania para diseñar este instrumento con el que se culminaría la Unión Bancaria, un mes después la posición del responsable de Finanzas en el gobierno alemán y principal valedor del acuerdo, Olaf Scholz, se había debilitado en el seno del ejecutivo germano tras perder la carrera por liderar el partido socialdemócrata alemán, SPD.

Las nuevas exigencias planteadas por Berlín (garantías adicionales, o también llamados «colchones de capital», exigidos a los bancos que muestren una excesiva sobreexposición de deuda) fueron recibidas como inasumibles por parte de los países del sur.

\section{Cuestiones generales de la actualidad económica}

\section{BCE: rebaja en los tipos de interés de depósito y nuevo programa de compra de deuda}

En Septiembre, el consejo de gobierno del BCE anunció un nuevo paquete para estimular la Economía de la Eurozona que incluía el incremento de los tipos negativos de la facilidad de depósito desde el $-0,4 \%$ al $-0,5 \%$, medida que pretende, en efecto, promover el crédito en la Eurozona, ante la perspectiva que se plantea a los bancos de soportar un mayor coste por depositar sus excesos de liquidez en el BCE.

En todo caso, y para contrarrestar en cierta medida los efectos negativos de esta decisión en el sector bancario, el Banco también acordó mejorar las condiciones aplicadas al exceso de las reservas depositadas por los bancos en Fráncfort, de tal manera que a partir del 30 de Octubre este exceso de reservas, siempre que no supere en más de seis veces el volumen 
de reservas obligatorias, no se ve afectado por la penalización de la tasa de depósito del $-0,5 \%$.

Y, por otra parte, el BCE también anunció en Septiembre el reinicio del programa de compra de deuda que había finalizado en Diciembre de 2018. La reactivación se realizaría por un valor de 20.000 millones de euros mensuales a partir del 1 de Noviembre, y se prolongaría durante el tiempo «que fuera necesario».

Esta indeterminación en el plazo previsto para la compra de bonos se vio acompañada por la afirmación en el comunicado que siguió a la reunión del BCE de que la época de tipos de interés ultrabajos iba a a alargarse más de lo esperado, «hasta que se aprecie que la perspectiva de inflación converge de forma robusta a un nivel inferior pero cercano al $2 \%$. Esta afirmación fue particularmente destacada por los medios financieros ya que venía a querer decir que, en efecto, la primera subida de tipos no se producirá previsiblemente a mediados de 2020, tal como el Banco había asegurado hasta entonces, sino que se aplazaba sine die hasta que se constate un incremento suficiente en el nivel de precios.

En todo caso, este nuevo paquete de medidas encaminadas a contrarrestar la debilidad económica de la Eurozona y la incertidumbre provocada por las tensiones comerciales internacionales, dejan aún más mermada la capacidad de actuación del BCE, y el propio presidente Draghi hizo un llamamiento a los «países con margen fiscal» para que también intervinieran y colaborasen en la reactivación económica de la Eurozona.

\section{Inicio del «Semestre Europeo 2020»}

La nueva Comisión Europea, presidida por Ursula Von der Leyen, hizo públicos el 17 de Diciembre los documentos con los que se iniciaba el ciclo del «Semestre Europeo 2020» de coordinación de las políticas económicas, presupuestarias y sociales.

El primer texto, titulado «Estrategia Anual de Crecimiento Sostenible», EACS, establece la estrategia de política económica y de empleo de la UE, situando la sostenibilidad y la inclusión social en el centro de dicha política económica.

El objetivo marcado es garantizar que Europa siga teniendo los sistemas de protección social más avanzados del mundo, se convierta en el primer continente climáticamente neutro y sea un polo dinámico de innovación y emprendimiento competitivo. En términos más generales, la EACS deberá facilitar el camino a la UE y a sus Estados miembros para alcanzar los Objetivos de Desarrollo Sostenible, ODS, de las Naciones 
Unidas, y que la Comisión ha integrado por primera vez en el Semestre Europeo.

La EACS de 2020 abarca cuatro dimensiones interrelacionadas y mutuamente reforzadas, que deben guiar las reformas estructurales, las políticas de empleo, las inversiones y las políticas presupuestarias responsables de todos los Estados miembros para lograr una economía al servicio de las personas y del planeta.

Estas dimensiones son:

- la sostenibilidad medioambiental: orientaciones en los ámbitos en que resulten más necesarias las reformas estructurales y las inversiones en pro de un modelo económico sostenible,

- el aumento de la productividad: orientaciones sobre las inversiones y las reformas estructurales que estimulen la investigación y la innovación, mejoren el acceso a la financiación y el funcionamiento de los mercados de productos y servicios, y eliminen los cuellos de botella en el entorno empresarial,

- la equidad: aplicación de políticas sociales que garanticen unas condiciones de trabajo justas para todos y que permitan a las personas adaptarse a circunstancias cambiantes en un período de grandes transformaciones,

- la estabilidad macroeconómica: respeto de las normas presupuestarias, aprovechando la flexibilidad de que están dotadas y completando la Unión Económica y Monetaria europea (UEM).

Por su parte, el segundo documento presentado en Diciembre es el llamado «Informe del Mecanismo de Alerta», IMA, que en esta ocasión recomendaba que trece Estados miembros se sometan a un Examen Exhaustivo en 2020 para determinar y evaluar la gravedad de sus desequilibrios macroeconómicos: Alemania, Bulgaria, Croacia, Chipre, España, Francia, Grecia, Irlanda, Italia, Países Bajos, Portugal, Rumanía y Suecia.

Y un tercer texto, titulado «Recomendación sobre la política económica de la zona del euro» instaba a los Estados miembros de la Eurozona a que adopten medidas para lograr un crecimiento integrador y sostenible, y a que impulsen la competitividad. También reclamaba políticas presupuestarias diferenciadas, así como una mayor coordinación en el marco del Eurogrupo en caso de empeoramiento de las perspectivas. La Recomendación también abogaba por más avances en la profundización de la Unión Económica y Monetaria, en particular mediante la realización de la Unión Bancaria y la Unión de los Mercados de Capitales, lo que también contribuiría a reforzar el papel internacional del euro. 


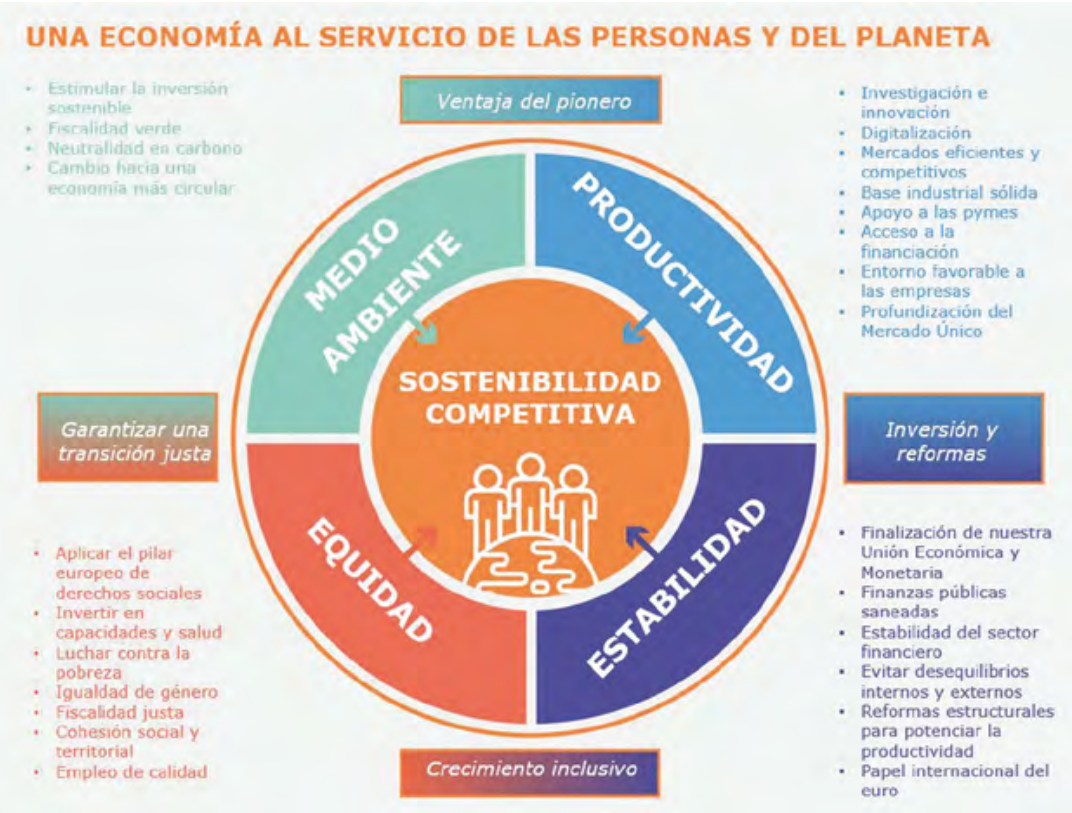

Estrategia anual de crecimiento sostenible 2020

Fuente: Comisión Europea

https://ec.europa.eu/transparency/regdoc/rep/1/2019/ES/COM-2019-650-F1-ES-MAIN-PART-1.PDF

\section{Guerra comercial con Estados Unidos: nuevos aranceles sobre productos europeos}

En el mes de Octubre se abría un nuevo capítulo en las hostilidades comerciales entre Estados Unidos y la Unión Europea, como consecuencia de una resolución de la Organización Mundial del Comercio que autorizaba a Washington a aplicar aranceles sobre las importaciones procedentes de la UE por valor de 7.500 millones de dólares, unos 6.900 millones de euros, para compensar los daños sufridos por la compañía aeronáutica norteamericana Boeing a causa de las ayudas y subsidios que diferentes gobiernos europeos habían concedido a su competidora Airbus para el desarrollo de sus aviones A350 y A380.

EE UU ya había presentado la primera queja por las ayudas de la UE a Airbus en 2004, y aunque algunos de sus argumentos fueron rechazados, la OMC sí concluyo que los gobiernos europeos habían concedido a Airbus créditos para el desarrollo de sus nuevos aviones a unos tipos de interés muy inferiores a los existentes en el mercado, por lo que crearon una ven- 
taja competitiva artificial a favor del consorcio europeo en el segmento de los grandes aviones de doble pasillo como el A350 y el A380, en perjuicio de su gran competidora en EE UU, Boeing.

Los aranceles norteamericanos «compensatorios» debían aplicarse sobre productos procedentes de Francia, Alemania, España y el Reino Unido, por ser éstos los cuatro países responsables de los subsidios ilegales, y en la lista de productos afectados figuraban diferentes componentes del sector de la aviación civil y aviones como los fabricados por Airbus, pero también un listado de productos agroalimentarios como quesos, vinos, aceites y licores.

El Gobierno español estimó en unos 756 millones de euros por el impacto de estos aranceles, como consecuencia de la pérdida de competitividad de las exportaciones de productos agroalimentarios españoles en el mercado estadounidense (fundamentalmente queso, vino y aceite de oliva).

La Comisión Europea mostró su preocupación por la reavivación de este largo conflicto que, en efecto, dura ya más de 15 años y considerando que, además, es un conflicto de doble sentido, puesto que la UE también se encuentra a la espera de que la OMC avale que puede aplicar medidas arancelarias similares a Estados Unidos por las ayudas que este país también concedió a Boeing.

De hecho, Bruselas tiene ya preparada una lista de productos (bebidas, alimentos, marroquinería, joyería y productos aeronáuticos) a los que aplicar aranceles por un valor de cerca de 20.000 millones de euros, aunque la cifra final dependerá de la decisión del arbitraje de la OMC.

La intención de Bruselas es no verse forzada a imponer estas tarifas, ya que si llegara a hacerlo, podría significar una nueva escalada en las tensiones con Estados Unidos, teniendo en cuenta que Donald Trump ha amenazado ya en varias ocasiones con aplicar aranceles a los automóviles y a sus componentes producidos en la UE alegando razones de «seguridad nacional».

Tras la entrada en vigor de los aranceles americanos el 18 de Octubre, la comisaria de Comercio, Cecilia Malmström, señaló que lamentaba «la elección que ha hecho EEUU de seguir adelante con los aranceles», añadiendo que «este paso no nos deja otra alternativa que seguir en su debido momento con nuestros propios aranceles por el caso Boeing, en el que EEUU ha sido declarado culpable de vulnerar las normas de la Organización Mundial del Comercio».

\section{Competencia: la Comisión investiga a España por ayudas ilegales a Peugeot}

La Comisión Europea anunció el 1 de Julio el inicio de una investigación para determinar si 20,7 millones de euros de fondos europeos procedentes del FEDER, destinados por España a la planta de Vigo de la com- 
pañía automovilística francesa Peugeot incumplen las normas comunitarias sobre ayudas de Estado de finalidad regional.

Aunque la normativa europea permite dedicar fondos públicos a impulsar la inversión privada, éstas están sujetas a una estricta condicionalidad, para evitar que los países falseen la competencia en el mercado europeo al beneficiar a unas determinadas empresas en perjuicio de las competidoras de estas empresas localizadas en otros Estados miembros.

«La inversión pública es importante para fomentar el crecimiento económico de las regiones desfavorecidas en Europa, pero debemos evitar la distorsión de la competencia entre los Estados miembros», señaló entonces la comisaria de Competencia, Margrethe Vestager.

En cambio, por parte del Grupo PSA, propietario de las marcas Peugeot, Citroën y Opel, se trató de restar importancia al tema al considerar, en particular, que las ayudas no habían sido desembolsadas y que, además, los fondos cuestionados estaban destinados a apoyar «inversiones de marcado carácter innovador» para transformar la planta gallega.

En cualquier caso, el ejecutivo comunitario deberá chequear si el dinero público destinado a la planta viguesa tiene un efecto incentivador, si se limita al mínimo para conseguir el objetivo de atraer la inversión, y si no provoca que la empresa traslade expresamente su producción desde otro país de la UE igual o más desfavorecido.

De hecho, Bruselas ya hizo públicas sus principales sospechas respecto a las ayudas públicas investigadas. Sus dudas se centraban en la posibilidad de que estas ayudas pudieran perjudicar a una región más desfavorecida de otro país europeo, en el hecho de que Peugeot hubiera realizado la inversión en Vigo sin necesidad de recibir las citadas ayudas, y sobre su contribución al desarrollo regional, su proporcionalidad o su efectividad en el fomento de la innovación. 


\section{Derechos de autor}

Los derechos de autor (para la distribución, comunicación pública, reproducción e inclusión en bases de datos de indexación y repositorios institucionales) de esta publicación (Cuadernos Europeos de Deusto, CED) pertenecen a la editorial Universidad de Deusto. El acceso al contenido digital de cualquier número de Cuadernos Europeos de Deusto es gratuito inmediatamente después de su publicación. Los trabajos podrán leerse, descargarse, copiar y difundir en cualquier medio sin fines comerciales y según lo previsto por la ley; sin la previa autorización de la Editorial (Universidad de Deusto) o el autor. Así mismo, los trabajos editados en CED pueden ser publicados con posterioridad en otros medios o revistas, siempre que el autor indique con claridad y en la primera nota a pie de página que el trabajo se publicó por primera vez en CED, con indicación del número, año, páginas y DOI (si procede). Cualquier otro uso de su contenido en cualquier medio o formato, ahora conocido o desarrollado en el futuro, requiere el permiso previo por escrito del titular de los derechos de autor.

\section{Copyright}

Copyright (for distribution, public communication, reproduction and inclusion in indexation databases and institutional repositories) of this publication (Cuadernos Europeos de Deusto, CED) belongs to the publisher University of Deusto. Access to the digital content of any Issue of Cuadernos Europeos de Deusto is free upon its publication. The content can be read, downloaded, copied, and distributed freely in any medium only for non-commercial purposes and in accordance with any applicable copyright legislation, without prior permission from the copyright holder (University of Deusto) or the author. Thus, the content of CED can be subsequently published in other media or journals, as long as the author clearly indicates in the first footnote that the work was published in CED for the first time, indicating the Issue number, year, pages, and DOI (if applicable). Any other use of its content in any medium or format, now known or developed in the future, requires prior written permission of the copyright holder. 